\title{
Asymptotic Formulas and Generalized Dedekind Sums
}

\author{
Gert Almkvist
}

\section{CONTENTS}

\section{Introduction}

2. Plane Partitions

3. Method A: The Abel-Plana Formula

4. Method B: Divergent Series

5. Method C: The Mellin Transformation

6. Generalized Dedekind Sums

7. A Ramanujan-Style Computation of a "Rather Exact" Asymptotic Formula

8. Method D: Eisenstein Series

9. Plane Partitions, the Fifth and Sixth terms

10. Triangular Partitions

11. The Long History of Kinkelin's Constant

\section{Conclusion}

Acknowledgements

References
We find asymptotic formulas as $\mathrm{n} \rightarrow \infty$ for the coefficients $a(r, n)$ defined by

$$
\prod_{\nu=1}^{\infty}\left(1-x^{\nu}\right)^{-\nu^{r}}=\sum_{n=0}^{\infty} a(r, n) x^{n} .
$$

(The case $r=1$ gives the number of plane partitions of $n$.) Generalized Dedekind sums occur naturally and are studied using the Finite Fourier Transform. The methods used are unorthodox; many of the computations are not justified but the result is in many cases very good numerically. The last section gives various formulas for Kinkelin's constant.

\section{INTRODUCTION}

In [Almkvist 1993] I gave the first four terms of an asymptotic formula for $\pi(n)$, the number of plane partitions of $n$. A form for higher-order terms is suggested there. This is false in general (beginning with $k=5$ ) and this paper describes a way to repair these errors.

More generally, let

$$
f(x)=\prod_{\nu=1}^{\infty}\left(1-x^{\nu}\right)^{-\nu^{r}}=\sum_{n=0}^{\infty} a(r, n) x^{n}
$$

(hence $\pi(n)=a(1, n)$ and $p(n)=a(0, n))$.

To find the $k$-th term in the asymptotic formula for $a(r, n)$ it is necessary to find very good estimates of $f(x)$ near the singular points $x=$ $\exp \left(\frac{2 \pi i h}{k}\right)$ with $(h, k)=1$. This is done by four different methods in Sections 3, 4, 5 and 8. Thus we find the expressions for

$$
f\left(\exp \left(\frac{2 \pi i h}{k}-t\right)\right)
$$

for small positive $t$. 
Then, in Section 7, comes the experimental part of the paper. To find $a(r, n)$ we put $x=\exp (i \varphi)$ in $f(x)$ and integrate on the unit circle (viewing $f(x)$ as a distribution). Then we extend the interval of integration from $[-\pi, \pi]$ to $(-\infty, \infty)$ assuming that the approximation (1-1) is valid on the entire imaginary axis. We have converted a Fourier coefficient to a Fourier transform. This hilarious computation gives in many cases very good asymptotic formulas. This is remarkable and I have no explanation why it works so often. For example, if $r=0$ we get

$$
a(0, n)=p(n),
$$

the number of partitions of $n$. The method above gives the Bessel function $I_{-3 / 2}$ instead of $I_{3 / 2}$ in the celebrated formula of Hardy, Ramanujan and Rademacher. The difference is very small.

As another example we obtain all 28 digits of $a(2,100)$ correctly, but "only" 46 out of 65 for $a(2,300)$. Most of the missing digits are recovered by using a pseudodifferential operator containing $\exp \left(-c D^{-1}\right)$ with $D=\frac{d}{d n}$. This is done in Section 8 where an Eisenstein series is used to find an approximate functional equation for $f\left(\exp \left(\frac{2 \pi i h}{k}-t\right)\right)$.

In Section 6 we study generalized Dedekind sums using the Finite Fourier Transformation.

In Section 9 the fifth and sixth terms of the asymptotic formula for $\pi(n)$ are computed. Numerically we get an error of only one or two digits out of 28 for $\pi(199)$ and $\pi(200)$.

In Section 10 we use our method to find exact formulas for the number of triangular partitions. My student Göran Andersson has shown that the method always gives the correct result if the generating function is rational (i.e., partitions into at most $r$ parts).

Finally, Section 11 briefly discusses the various definitions of Kinkelin's constant, first studied in 1860. The simplest one, found recently by Vardi and Meurman, is

$$
K=\zeta^{\prime}(-1),
$$

where $\zeta(s)$ is the Riemann zeta function.

\section{PLANE PARTITIONS}

Let $\pi(n)$ denote the number of plane partitions of $n$. Thus $\pi(3)=6$, since we have the following patterns (see [Andrews 1976] for an exact definition):

$\begin{array}{llllll}3 & 21 & 2 & 111 & 1 & 11 \\ & & 1 & & & 1 \\ & & & & & 1 \\ & & & & & \end{array}$

By convention, $\pi(0)=1$. Major MacMahon showed that the generating function for $\pi$ is

$$
\sum_{0}^{\infty} \pi(n) x^{n}=\prod_{\nu=1}^{\infty}\left(1-x^{\nu}\right)^{-\nu} .
$$

This is much more difficult than the corresponding result of Euler for ordinary partitions [Andrews 1976].

The value of $\pi(n)$ grows very fast with $n$. E. M. Wright [1931] showed that

$$
\pi(n) \sim \frac{e^{K} a^{7 / 36}}{2 \sqrt{\pi}}\left(\frac{2}{n}\right)^{25 / 36} \exp \left(3\left(a n^{2} / 4\right)^{1 / 3}\right),
$$

where

$$
\begin{aligned}
a & =\zeta(3)=\sum_{1}^{\infty} \frac{1}{k^{3}}, \\
K & =2 \int_{0}^{\infty} \frac{x \log x}{e^{2 \pi x}-1} d x .
\end{aligned}
$$

C. Knessl [1990] found a second term of size approximately equal to the square root of Wright's term. For the actual computation of $\pi(n)$ this is rather useless since the error in the first term is much larger than the second term. This depends on the fact that the exponential is not the correct function for approximating $\pi(n)$. In [Almkvist 1993] the following formula

$$
\pi(n) \approx \varphi_{1}(n)+\varphi_{2}(n)+\cdots
$$

is given, where

$$
\begin{aligned}
& \varphi_{1}(n)=e^{K} a^{13 / 24} \\
& \times \exp \left(-\sum_{i=1}^{\infty} \frac{2(2 i+1) ! \zeta(2 i) \zeta(2 i+2)}{i(2 \pi)^{4 i+2}} D^{2 i}\right) g\left(n \sqrt{a}, \frac{-1}{12}\right)
\end{aligned}
$$


with

$$
D=\frac{d}{d n}
$$

and

$$
g(x, \gamma)=\sum_{\nu=0}^{\infty} \frac{x^{2 \nu+\gamma-1}}{\nu ! \Gamma(2 \nu+\gamma)} .
$$

Formulas for $\varphi_{2}, \varphi_{3}$ and $\varphi_{4}$ are also given. The form for higher-order terms is suggested. This is false in general (starting at $k=5$ ). The constant $c_{k}$ depends on $h$ (the $k$-th term corresponds to the behaviour of $\prod\left(1-x^{\nu}\right)^{-\nu}$ at the singular point $\exp (2 \pi i h / k))$. This paper describes the author's work to repair these errors. It will lead to many new problems, some of them, possibly more interesting than the original problem. To begin with we generalize the problem to

$$
f(x)=\prod_{\nu=1}^{\infty}\left(1-x^{\nu}\right)^{-\nu^{r}}=\sum_{n=0}^{\infty} a(r, n) x^{n} .
$$

Thus

$a(0, n)=p(n)=$ number of partitions of $n$, $a(1, n)=\pi(n)=$ number of plane partitions of $n$.

To find the asymptotic behaviour of $a(r, n)$ as $n \rightarrow$ $\infty$ it is necessary to study $f(x)$ near its singular points $x=\exp (2 \pi i h / k)$, where $(h, k)=1$. This is achieved by substituting

$$
x=\exp (2 \pi i h / k-t)
$$

and letting $t \rightarrow 0$. The main term will come from the point $x=1$ and we put $x=e^{-t}$.

We will now describe four different methods (labelled A, B, C, D) to find the expansion of

$$
f(\exp (2 \pi i h / k-t))
$$

as $t \rightarrow 0$.

\section{METHOD A: THE ABEL-PLANA FORMULA}

This great formula, which seems to be almost completely forgotten, is a concise version of the EulerMaclaurin summation formula. If $h(z)$ is a function behaving nicely at $\infty$ (see [Henrici 1974, p. 274] for a precise statement), then

$$
\begin{aligned}
\sum_{0}^{\infty} h(n)=\int_{0}^{\infty} h(x) d x & +\frac{h(0)}{2} \\
& +i \int_{0}^{\infty} \frac{h(i y)-h(-i y)}{e^{2 \pi y}-1} d y .
\end{aligned}
$$

We want to study

$$
g(t)=\log f\left(e^{-t}\right)=-\sum_{\nu=1}^{\infty} \nu^{r} \log \left(1-e^{-\nu t}\right) .
$$

Hence put

$$
h(x)=-x^{r} \log \left(1-e^{-t x}\right)
$$

in the Abel-Plana formula

$$
\begin{aligned}
& g(t)=-\int_{0}^{\infty} x^{r} \log \left(1-e^{-t x}\right) d x \\
& \quad-i \int_{0}^{\infty} \frac{(i y)^{r} \log \left(1-e^{-i t y}\right)-(-i y)^{r} \log \left(1-e^{i t y}\right)}{e^{2 \pi y}-1} d y .
\end{aligned}
$$

Expanding $\log \left(1-e^{-t z}\right)$ in Taylor series and using

$$
\int_{0}^{\infty} \frac{y^{\nu}}{e^{2 \pi y}-1} d y=\frac{\Gamma(\nu+1) \zeta(\nu+1)}{(2 \pi)^{\nu+1}}=-\frac{\zeta(-\nu)}{2 \sin (\nu \pi / 2)}
$$

(and assuming for the moment that $\nu$ is not an integer) we get

$$
\zeta(-\nu)=-2 \sin \frac{\nu \pi}{2} \int_{0}^{\infty} \frac{y^{\nu}}{e^{2 \pi y}-1} d y
$$

and

$$
\begin{aligned}
\zeta^{\prime}(-\nu)=\pi \cos \frac{\nu \pi}{2} \int_{0}^{\infty} \frac{y^{\nu}}{e^{2 \pi y}-1} d y & \\
& +2 \sin \frac{\nu \pi}{2} \int_{0}^{\infty} \frac{y^{\nu} \log y}{e^{2 \pi y}-1} d y .
\end{aligned}
$$

Hence

$$
\begin{aligned}
g(t)=\frac{r ! \zeta(r+2)}{t^{r+1}}+\zeta^{\prime}(-r)-\zeta(-r) \log t \\
\quad+\frac{t}{2} \zeta(-1-r)+\sum_{\nu=2}^{\infty} \frac{\zeta(1-\nu) \zeta(-r-\nu)}{\nu !} t^{\nu}
\end{aligned}
$$


In Section 7 we will show how we can find the first approximation $\varphi_{1}(n)$ of $a(r, n)$ using the formula for $g(t)$ above. Using the functional equation

$$
f(-x)=\frac{f\left(x^{2}\right)^{2^{r+1}+1}}{f(x) f\left(x^{4}\right)^{2^{r}}}
$$

for

$$
f(x)=\prod_{\nu=1}^{\infty}\left(1-x^{\nu}\right)^{-\nu^{r}},
$$

we can easily obtain the second term corresponding to the singular point $x=-1$. However, it seems hard to find the higher-order terms, corresponding to $x=\exp (2 \pi i h / k)$, by this method.

\section{METHOD B: DIVERGENT SERIES}

This "method" should be used with care. It is easy to lose some terms. It is mentioned here since it was in this way that I found the generalized Dedekind sums.

We wish to study $f(x)=\prod_{n=1}^{\infty}\left(1-x^{\nu}\right)^{-\nu^{r}}$ near $x=\exp (2 \pi i h / k)$. Hence we put

$$
x=\exp (2 \pi i h / k-t)
$$

and take the logarithm

$$
\begin{aligned}
g(t)= & \log f(\exp (2 \pi i h / k-t)) \\
= & -\sum_{\nu=1}^{\infty} \nu^{r} \log (1-\exp (2 \pi i \nu h / k-\nu t)) \\
= & \sum_{\nu=1}^{\infty} \sum_{\mu=1}^{\infty} \frac{\nu^{r}}{\mu} e^{-\mu \nu t} \exp (2 \pi i h \mu \nu / k) \\
= & \frac{1}{k} \sum_{\nu=1}^{\infty} \sum_{q=1}^{\infty} \frac{\nu^{r}}{q} e^{-q \nu(k t)} \\
& +\sum_{\nu=1}^{\infty} \sum_{j=1}^{k-1} \sum_{q=0}^{\infty} \frac{\nu^{r}}{q k+j} e^{-(k q+j) \nu t} \exp (2 \pi i j \nu h / k) \\
= & S_{1}+S_{2} .
\end{aligned}
$$

The summand $S_{1}=\frac{1}{k} f\left(e^{-k t}\right)$ is known by the Abel-Plana formula. In order to compute $S_{2}$ we first sum over $q$.
Lemma 4.1. Let $1 \leq j<q$. Then

$$
\begin{aligned}
\sum_{q=0}^{\infty} \frac{e^{-(k q+j) u}}{k q+j}=-\frac{1}{k} & \left(\log u-\log k-\frac{\pi}{2} \cot \frac{j \pi}{k}\right. \\
& +\sum_{\mu=1}^{k-1} \cos \frac{2 \mu j \pi}{k} \log \left(2 \sin \frac{\mu \pi}{k}\right) \\
& \left.+\sum_{\nu=1}^{\infty} \frac{(-1)^{\nu} k^{\nu} B_{\nu}(j / k)}{\nu \nu !} u^{\nu}\right)
\end{aligned}
$$

where $B_{\nu}(x)$ is the Bernoulli polynomial of degree $\nu$.

The proof uses the value of $\psi(j / k)$ (where $\psi(x)=$ $\left.\Gamma^{\prime}(x) / \Gamma(x)\right)$, which was known to Gauss. We get

$$
\begin{aligned}
S_{2}=\frac{1}{k} \sum_{\nu=1}^{\infty} & \sum_{j=1}^{k-1} \nu^{r} \exp (2 \pi i j \nu h / k) \\
& \times\left(-\log t-\log \nu-\log k+\frac{\pi}{2} \cot \frac{j \pi}{k}\right. \\
& +\sum_{\mu=1}^{k-1} \exp (2 \pi \mu j i / k) \log \left(2 \sin \frac{\mu \pi}{k}\right) \\
& \left.-\sum_{\mu=1}^{\infty} \frac{(-1)^{\mu} k^{\mu} B_{\mu}(j / k) \nu^{\mu}}{\mu \mu !} t^{\mu}\right) .
\end{aligned}
$$

Then, summing like Euler, we get

$$
\begin{gathered}
\sum_{1}^{\infty} \nu^{r}=\zeta(-r), \\
\sum_{1}^{\infty} \nu^{r} \log \nu=-\zeta^{\prime}(-r) .
\end{gathered}
$$

By formally differentiating

$$
\sum_{1}^{\infty} e^{i \nu \alpha}=\frac{1}{2}(i \cot (\alpha / 2)-1)
$$

$r$ times we get

$$
\sum_{1}^{\infty} \nu^{r} e^{i \nu \alpha}=\frac{i}{2(2 i)^{r}} \cot ^{(r)}(\alpha / 2)
$$


This gives rise to a very interesting term of $S_{2}$ (for even $r$ ), namely

$$
\begin{aligned}
\frac{\pi}{2 k} \sum_{j=1}^{k-1} \cot \frac{j \pi}{k} \sum_{\nu=1}^{\infty} \nu^{r} \exp \left(\frac{2 \pi i j \nu h}{k}\right) \\
=\pi i \frac{1}{4 k(2 i)^{r}} \sum_{j=1}^{k-1} \cot \left(\frac{j \pi}{k}\right) \cot ^{(r)}\left(\frac{j h \pi}{k}\right) \\
=\pi i s(r, h, k)
\end{aligned}
$$

where

$$
s(r, h, k)=\frac{1}{4 k(2 i)^{r}} \sum_{j=1}^{k-1} \cot \left(\frac{j \pi}{k}\right) \cot ^{(r)}\left(\frac{j h \pi}{k}\right)
$$

is a generalized Dedekind sum.

Indeed,

$$
s(0, h, k)=\frac{1}{4 k} \sum_{j=1}^{k-1} \cot \left(\frac{j \pi}{k}\right) \cot \left(\frac{j h \pi}{k}\right),
$$

which is equal to the classical Dedekind sum,

$$
s(h, k)=\sum_{j=1}^{k-1}((j / k))((j h / k))
$$

where

$$
((x))= \begin{cases}x-[x]-\frac{1}{2} & \text { if } x \notin \mathbb{Z}, \\ 0 & \text { if } x \in \mathbb{Z} .\end{cases}
$$

The equality of these two expressions for $s(h, k)$ is usually attributed to Rademacher [1933]. But the cotangent formula already appears in [Mellin 1923], in a formula that approximates $\prod_{1}^{\infty}\left(1-x^{\nu}\right)^{-1}$ near $x=\exp (2 \pi i h / k)$.

Summing the other terms of $S_{2}$ we get a formula for $\log f(\exp (2 \pi i h / k-t))$, which is close to the correct one; but we are of course far from a proof. In the next section we will use a safer method.

\section{METHOD C: THE MELLIN TRANSFORMATION}

This is by far the best method and we can even prove an asymptotic formula for $f(\exp (2 \pi i h / k-t))$ as $t \rightarrow 0+$.
Theorem 5.1. Let $f(x)=\prod_{\nu=1}^{\infty}\left(1-x^{\nu}\right)^{-\nu^{r}}$.

(i) Assume $r$ is even $\geq 2$. Then, as $t \rightarrow 0+$,

$$
\begin{aligned}
& \log f(\exp (2 \pi i h / k-t))=\frac{r ! \zeta(r+2)}{k^{r+2}} \frac{1}{t^{r+1}} \\
& \quad+k^{r} \zeta^{\prime}(-r)+\pi i s(r, h, k)+\frac{1}{2} \zeta(-1-r) t
\end{aligned}
$$

where

$$
s(r, h, k)=\frac{k^{r}}{r+1} \sum_{j=1}^{k-1} B_{r+1}(j / k)((j h / k))
$$

is a generalized Dedekind sum (we use the same notation as in Section 4 , since these two $s(r, h, k)$ are identical; see Section 6 ). (If $r=0$ we get an extra term $-\zeta(0) \log t=\frac{1}{2} \log t$ in $(5-1)$.)

(ii) We have, if $r$ is odd,

$$
\begin{aligned}
& \log f(\exp (2 \pi i h / k-t)) \\
& =\frac{r ! \zeta(r+2)}{k^{r+2}} \frac{1}{t^{r+1}}+k^{r} \zeta^{\prime}(-r)-k^{r} \zeta(-r) \log k \\
& \quad+\frac{k^{r}}{r+1} \sum_{j=1}^{k-1} B_{r+1}(j / k) \log \left|2 \sin \frac{j h \pi}{k}\right| \\
& \quad-k^{r} \zeta(-r) \log t
\end{aligned}
$$

$$
\begin{aligned}
& +\frac{i k^{r+1}}{2(r+2)} \sum_{j=1}^{k-1} B_{r+2}(j / k) \cot \left(\frac{j h \pi}{k}\right) \\
& +\sum_{\nu=2}^{\infty} \frac{(-1)^{\nu} k^{\nu+r} t^{\nu}}{\nu \nu !(\nu+r+1)}\left(B_{\nu} B_{\nu+r+1}\right. \\
& \left.+\frac{\nu}{(2 i)^{\nu}} \sum_{j=1}^{k-1} B_{\nu+r+1}(j / k) \cot ^{(\nu-1)}\left(\frac{j h \pi}{k}\right)\right) .
\end{aligned}
$$

Sketch of proof. We compute

$$
\begin{aligned}
& g(t)=\log f(\exp (2 \pi i h / k-t)) \\
&=-\sum_{\nu=1}^{\infty} \nu^{r} \log (1-\exp (2 \pi i h \nu / k-\nu t)) \\
&=\sum_{\nu=1}^{\infty} \sum_{d=1}^{k} \sum_{\mu=0}^{\infty} \frac{\nu^{r}}{\mu k+d} \exp (-\nu(\mu k+d) t) \\
& \times \exp (2 \pi i \nu d h / k) .
\end{aligned}
$$


The Mellin transform

$$
\begin{aligned}
& \tilde{g}(s)= \int_{0}^{\infty} g(t) t^{s-1} d t \\
&= \sum_{d=1}^{k} \sum_{\nu=1}^{\infty} \sum_{\mu=0}^{\infty} \frac{\nu^{r-s} \Gamma(s)}{(\mu k+d)^{1+s}} \exp (2 \pi i \nu d h / k) \\
&=\Gamma(s) k^{-r-2 s-1} \sum_{d=1}^{k} \sum_{j=1}^{k} \zeta\left(1+\begin{array}{r}
s, d / k) \\
\times \zeta(-r+s, j / k) \\
\times \exp (2 \pi i j d h / k),
\end{array}\right.
\end{aligned}
$$

where

$$
\zeta(s, a)=\sum_{n=0}^{\infty} \frac{1}{(n+a)^{s}},
$$

is the Hurwitz $\zeta$-function.

To recover $g(t)$ we use Mellin's inversion formula

$$
g(t)=\frac{1}{2 \pi i} \int_{a-i \infty}^{a+i \infty} \tilde{g}(s) t^{-s} d s
$$

where $a>1+r$. We complete the path of integration to a rectangle where the left vertical side goes through $-N-1 / 2$ where $N$ is a large integer.

The main term of the expansion of $g(t)$ near $t=$ 0 will be

$$
\frac{r ! \zeta(r+2)}{k^{r+2}} \frac{1}{t^{r+2}}
$$

which is the residue of $\tilde{g}(s) t^{-s}$ at the simple pole $s=r+1$.

The most difficult part is the calculation of the residue at $s=0$, a double pole. Expanding everything at $s=0$ and deleting everything of order $s^{2}$ we get

$$
\begin{aligned}
\tilde{g}(s) t^{-s}= & k^{r-1}(1-2 s \log k)(1-s \log t) \\
\times \sum_{d=1}^{k} \sum_{j=1}^{k}\left(s^{-2}-s^{-1}(\gamma+\psi\right. & (j / k))) \\
\times(\zeta(-r)+ & \left.s \zeta^{\prime}(-r, j / k)\right) \\
& \times \exp (2 \pi i j d h / k),
\end{aligned}
$$

where $\gamma$ is Euler's constant and $\psi(x)=\Gamma^{\prime}(x) / \Gamma(x)$ as before. Using

$$
\begin{aligned}
\sum_{j=1}^{k-1} \log \left(2 \sin \frac{j \pi}{k}\right) & =\log k, \\
\sum_{j=1}^{k-1} \cot \left(\frac{j \pi}{k}\right) \exp \left(\frac{2 \pi i j \mu}{k}\right) & =\frac{2 k}{i}((\mu / k))
\end{aligned}
$$

[Rademacher and Grosswald 1972, p. 14], we get, after some tricky computations,

$$
\begin{aligned}
& \operatorname{Res}_{s=0}\left(\tilde{g}(s) t^{-s}\right) \\
& =k^{r} \zeta(-r) \log (k t)+k^{r} \zeta^{\prime}(-r)+\pi i s(r, h, k) \\
& \quad+\frac{k^{r}}{r+1} \sum_{j=1}^{k-1} B_{r+1}(j / k) \log \left|2 \sin \left(\frac{j h \pi}{k}\right)\right| .
\end{aligned}
$$

The other terms are obtained as the residues at $s=-\nu$ for $\nu=1,2,3, \ldots$.

Using the Riemann-Lebesgue Lemma one can show that the integral on the other three sides tends to zero as the rectangle goes to infinity and $t \rightarrow 0+$.

\section{GENERALIZED DEDEKIND SUMS}

In Section 5, when we estimated

$$
f(x)=\prod_{\nu=1}^{\infty}\left(1-x^{\nu}\right)^{-\nu^{r}}
$$

at the singular point $\exp (2 \pi i h / k)$, we found formulas containing the functions $\zeta(-\nu, x), \cot ^{(\nu)}(\pi x)$, $\log |2 \sin (\pi x)|,((x))$, and $B_{r+1}(x)$. What is common to all these functions? They all satisfy a functional equation ("addition theorem") of the type

$$
\sum_{j=0}^{k-1} f\left(\frac{x+j}{k}\right)=k^{m} f(x),
$$

where $m$ is a particular integer and $k>1$ is any integer. We call such a function a Kubert function of type $m$. Let $K_{m}$ denote the vector space of all such functions satisfying (6-1). (In [Milnor 1983] a different notation is used.) 
Let's order these functions, and a few others, according to their type:

$$
\begin{array}{rl}
m & f \\
3 & \zeta(3, x), \zeta(3,1-x), \cot ^{(2)}(\pi x) \\
2 & \zeta(2, x), \zeta(1-x), \cot ^{(1)}(\pi x) \\
1 & 1, \cot (\pi x) \\
0 & B_{1}(x),((x)), \log |2 \sin (\pi x)| \\
-1 & B_{2}(x), \Lambda(\pi x), \ell(2, x), \ell(2,1-x) \\
-2 & B_{3}(x), \ell(3, x), \ell(3,1-x) \\
-3 & B_{4}(x), \ell(4, x), \ell(4,1-x)
\end{array}
$$

Here

$$
\Lambda(y)=-\int_{0}^{y} \log (2 \sin t) d t
$$

is the Lobachevsky function and

$$
\ell(s, x)=\sum_{n=1}^{\infty} \frac{\exp (2 \pi i n x)}{n^{s}}
$$

is the periodic $\zeta$-function.

Theorem 6.1 (Kubert). $K_{m}$ is two-dimensional for all $m$.

Thus there are linear relations like

$$
\cot ^{(m-1)}(\pi x)=C\left(\zeta(m, x)+(-1)^{m} \zeta(m, 1-x)\right)
$$

for some constant $C$

$\zeta(1-s, x)=\frac{\Gamma(s)}{(2 \pi)^{s}}\left(e^{-\pi i s / 2} \ell(s, x)+e^{\pi i s / 2} \ell(s, 1-x)\right)$

(Hurwitz's formula).

The Fourier expansion of $B_{r}(x)$ in the interval $(0,2 \pi)$ shows that $B_{r}(x)$ is a linear combination of $\ell(r, x)$ and $\ell(r, 1-x)$.

We also have

$$
\Lambda(\pi x)=\frac{i \pi^{2}}{2} B_{2}(x)-\frac{i}{2} \ell(2, x) .
$$

\section{The Finite Fourier Transformation (FFT)}

Consider periodic functions $f$ satisfying

$$
f(x+1)=f(x)
$$

and let $k>1$ be a fixed integer. We define the finite Fourier transform $\hat{f}$ of $f$ by

$$
\hat{f}(\mu / k)=\sum_{j=0}^{k-1} f(j / k) \exp (-2 \pi i j \mu / k) .
$$

We have the inverse transformation

$$
f(\nu / k)=\frac{1}{k} \sum_{\mu=0}^{k-1} \hat{f}(\mu / k) \exp (2 \pi i \mu \nu / k)
$$

so

$$
\hat{\hat{f}}(x)=k f(1-x) .
$$

We also define the scalar product

$$
\langle f, g\rangle=\sum_{j=0}^{k-1} f(j / k) \overline{g(j / k)}
$$

and obtain

$$
k\langle f, g\rangle=\langle\hat{f}, \hat{g}\rangle \quad \text { ("Parseval's formula"). }
$$

Here is a small table of FFT's:

$$
\begin{aligned}
f & \hat{f} \\
((x)) & \frac{i}{2} \cot (\pi x) \quad \text { (Eisenstein) } \\
\ell(s, x) & k^{1-s} \zeta(s, x) \\
B_{m}(x) & m k^{1-m}(i / 2)^{m} \cot ^{(m-1)}(\pi(x)) \\
\log (2 \sin (\pi x)) & \gamma+\log k+\frac{\pi}{2} \cot (\pi x)+\psi(x)
\end{aligned}
$$

By inspection $\left(\operatorname{dim} K_{m}=2\right)$ we see that, for all integers $m \neq 0$,

$$
\begin{aligned}
& f \in K_{m} \Longrightarrow \hat{f} \in K_{1-m}, \\
& f \in K_{m} \Longrightarrow f^{\prime} \in K_{m+1} .
\end{aligned}
$$

Definition 6.2. For positive integers $h, k$ with $k>1$ and functions $f$ and $g$ we define the Dedekind sum

$$
s(f, g ; h, k)=\sum_{j=0}^{k-1} f(j / k) g(j h / k) .
$$

Using Parseval's formula we obtain the following result: 
Proposition 6.3. $k s(f, g ; h, k)=s(\hat{g}, \hat{f} ; h, k)$.

Examples. (i) Let $f(x)=g(x)=((x))$. Then

$$
s(f, g ; h, k)=\sum_{j=0}^{k-1}((j / k))((j h / k))=s(h, k)
$$

is the classical Dedekind sum. Now

$$
\hat{f}(x)=\hat{g}(x)=\frac{i}{2} \cot (\pi x)
$$

and Proposition 6.3 shows that

$$
s(h, k)=\frac{1}{4 k} \sum_{j=1}^{k-1} \cot \left(\frac{j \pi}{k}\right) \cot \left(\frac{j h \pi}{k}\right)
$$

(Mellin-Rademacher); here we have used the fact that $\hat{f}(0)=f(0)=0$, by the functional equation.

(ii) Put $f(x)=B_{r+1}(x)$ and $g(x)=((x))$. Then we get, for even $r$,

$$
\begin{aligned}
s(r, h, k) & =\frac{k^{r}}{r+1} \sum_{j=1}^{k-1} B_{r+1}(j / k)((j h / k)) \\
& =\frac{1}{4 k(2 i)^{r}} \sum_{j=1}^{k-1} \cot ^{(r)}\left(\frac{j h \pi}{k}\right) \cot \left(\frac{j \pi}{k}\right),
\end{aligned}
$$

the formula referred to in Section 5 .

(iii) We have the Dedekind zeta function

$$
L(s)=\zeta_{Q \sqrt{-7}}(s)=\frac{1}{2} \sum_{\substack{m, n \in \mathbb{Z} \\(m, n) \neq(0,0)}}\left(m^{2}+m n+2 n^{2}\right)^{-s}
$$

of the field $\mathbb{Q}(\sqrt{-} 7)$ (see [Zagier 1986]). We want to compute $L(s)$ for integer $s$. By [Zucker and Robertson 1975] we have

$$
L(s)=7^{-s} \zeta(s) \sum_{\nu=1}^{6}\left(\frac{\nu}{7}\right) \zeta(s, \nu / 7),
$$

where $\left(\frac{\nu}{7}\right)$ is the Legendre symbol. We consider this as a Dedekind sum for $k=7$ with

$$
\begin{aligned}
f(\mu / 7) & =\left(\frac{\mu}{7}\right), \\
g\left(\frac{\mu}{7}\right) & =\zeta(s, \mu / 7) .
\end{aligned}
$$

Then

$$
\begin{aligned}
& \hat{f}(\mu / 7)=-i \sqrt{7}\left(\frac{\mu}{7}\right), \\
& \hat{g}(\mu / 7)=7^{s} \ell(s, 1-\mu / 7),
\end{aligned}
$$

and we obtain by Parseval's formula

$$
\begin{aligned}
L(s) & =7^{-s} \zeta(s) \cdot 7^{-1}\langle\hat{f}, \hat{g}\rangle \\
& =-\frac{i}{7} \zeta(s) \cdot \sqrt{7} \sum_{\mu=1}^{6}\left(\frac{\mu}{7}\right) \ell(s, 1-\mu / 7) \\
& =\frac{2 \zeta(s)}{\sqrt{7}}\left(\mathrm{Cl}_{s}\left(\frac{2 \pi}{7}\right)+\mathrm{Cl}_{s}\left(\frac{4 \pi}{7}\right)+\mathrm{Cl}_{s}\left(\frac{8 \pi}{7}\right)\right),
\end{aligned}
$$

where

$$
\mathrm{Cl}_{s}(x)=\sum_{n=1}^{\infty} \frac{\sin (n x)}{n^{s}}
$$

is the Clausen function. In particular for $s=2$ we get, since $\mathrm{Cl}_{2}(x)=2 \Lambda(2 x)$,

$$
\begin{aligned}
\zeta_{Q(\sqrt{-7})}(2) & =\frac{2 \pi^{2}}{3 \sqrt{7}}\left(\Lambda\left(\frac{4 \pi}{7}\right)+\Lambda\left(\frac{8 \pi}{7}\right)+\Lambda\left(\frac{16 \pi}{7}\right)\right) \\
& =\frac{2 \pi^{2}}{3 \sqrt{7}}(\Lambda(\pi / 7)+\Lambda(2 \pi / 7)+\Lambda(4 \pi / 7)) .
\end{aligned}
$$

\section{A RAMANUJAN-STYLE COMPUTATION OF A "RATHER EXACT" ASYMPTOTIC FORMULA}

According to Hardy, Ramanujan never mastered complex integration. But already in his first letter to Hardy in 1913 he states that the coefficient of $x^{n}$ in

$$
\frac{1}{1-2 x+2 x^{4}-2 x^{9}+2 x^{16} \mp \cdots}
$$

is the nearest integer to

$$
\frac{1}{4 n}\left(\cosh (\pi \sqrt{n})-\frac{\sinh (\pi \sqrt{n})}{\pi \sqrt{n}}\right) .
$$

As Selberg has pointed out, there occurs here

$$
\frac{d}{d n}\left(\frac{\sinh (\pi \sqrt{n})}{\sqrt{n}}\right) \text {. }
$$

This expression is absent in the Hardy-Ramanujan formula for $p(n)$, but reappears in Rademacher's convergent series. 
It is likely that Ramanujan used Fourier series and Fourier transformations to obtain such a formula.

Let's show how to find $a(r, n)$ when $r$ is even and greater than 2 , where

$$
f(x)=\prod_{\nu=1}\left(1-x^{\nu}\right)^{-\nu^{r}}=\sum_{0}^{\infty} a(r, n) x^{n} .
$$

Put $x=e^{i y}$ and compute the Fourier coefficent,

$$
a(r, n)=\frac{1}{2 \pi} \int_{-\pi}^{\pi} f\left(e^{i y}\right) e^{-i n y} d y .
$$

This might look dangerous, but $f\left(e^{i y}\right)$ is singular "only" for $y=2 \pi i h / k$ where $(h, k)=1$, a set of measure zero.

Then put

$$
y=2 \pi h / k+\varphi
$$

and assume that the asymptotic formula for $g(t)$ in Section 5 is valid also for $t=-i \varphi$, i.e.,

$$
\begin{aligned}
f(\exp (2 \pi i h / k+i \varphi)) & \sim \exp \left(\frac{a}{k^{r+2}}(-i \varphi)^{-(r+1)}\right. \\
+k^{r} \zeta^{\prime}(-r) & \left.+\pi i s(r, h, k)-\frac{\zeta(-1-r)}{2} i \varphi\right)
\end{aligned}
$$

(this is the most dubious part of this computation).

Let $\varphi_{k}(n)$ denote the contribution to the integral near the points $\exp (2 \pi i h / k)$ for all $h=1,2, \ldots, k$ with $(h, k)=1$. We also extend the integration to the interval $(-\infty, \infty)$ since most of the mass is concentrated near $\varphi=0$. Then

$$
\begin{aligned}
& \varphi_{k}(n) \\
& \sim \exp \left(k^{r} \zeta^{\prime}(-r)\right) \sum_{(h, k)=1} \exp (\pi i s(r, h, k)-2 \pi i h n / k) \\
& \quad \times \frac{1}{2 \pi} \int_{-\infty}^{\infty} \exp \left(\frac{a}{k^{r+2}(-i \varphi)^{r+1}}-\left(n+\frac{\zeta(-1-r)}{2}\right) i \varphi\right) d \varphi \\
& =\exp \left(k^{r} \zeta^{\prime}(-r)\right) A(r, k, n) \\
& \quad \times \frac{1}{2 \pi} \int_{-\infty}^{\infty} \exp \left(\frac{a(-i \varphi)^{-r-1}}{k^{r+2}}-i \xi \varphi\right) d \varphi
\end{aligned}
$$

where

$$
\begin{aligned}
a & =r ! \zeta(r+2), \\
\xi & =n+\frac{\zeta(-1-r)}{2}, \\
A(r, k, n) & =\sum_{(h, k)=1} \exp (\pi i s(r, h, k)-2 \pi i h n / k) .
\end{aligned}
$$

Now

$$
\exp \left(\frac{a(-i \varphi)^{-r-1}}{k^{r+2}}\right)=\sum_{\nu=0}^{\infty} \frac{a^{\nu}}{k^{\nu(r+2)} \nu !}(-i \varphi)^{-\nu(r+1)}
$$

and

$\frac{1}{2 \pi} \int_{-\infty}^{\infty}(-i \varphi)^{-\nu(r+1)} e^{-i \xi \varphi}= \begin{cases}\frac{\xi^{\nu(r+1)-1}}{(\nu(r+1)-1) !} & \text { if } \nu \geq 1 \\ \delta(\xi) & \text { if } \nu=0\end{cases}$

(as distributions).

Since $\xi>0$ we can delete the delta function and hence

$$
\begin{aligned}
& \varphi_{k}(n) \sim A(r, k, n) \exp \left(k^{r} \zeta^{\prime}(-r)\right) \\
& \times \sum_{\nu=1}^{\infty} \frac{a^{\nu} \xi^{\nu(r+1)-1}}{k^{\nu(r+2)} \nu !(\nu(r+1)-1) !} \\
&=A(r, k, n) \exp \left(k^{r} \zeta^{\prime}(-r)\right) \\
& \times \frac{(r+1) ! \zeta(r+2)}{k^{r+2}} L\left(r+1, \frac{a \xi^{r+1}}{k^{r+2}}\right),
\end{aligned}
$$

where

$$
L(m, x)=\sum_{\nu=0}^{\infty} \frac{x^{\nu}}{\nu !(m(\nu+1)-1) !}
$$

If

$$
y=x^{m} L\left(m, x^{m}\right)
$$

then

$$
x y^{(m+1)}=m y \text {. }
$$




\section{A Numerical Example}

Let $r=2$ and $n=100$. Then

$$
\begin{aligned}
& \varphi_{1}=2330211343210833803718557774.349, \\
& \varphi_{2}=\quad 3612679905487.906 \text {, } \\
& \varphi_{3}=\quad-40315672.924 \text {, } \\
& \varphi_{4}=\quad 158721.384, \\
& \varphi_{5}=\quad 6526.089, \\
& \varphi_{6}=\quad 245.983 \text {, } \\
& \varphi_{7}=\quad-46.124 \text {, }
\end{aligned}
$$

This compares with

$$
a(2,100)=2330211343210837416358313037,
$$

so the error is 0.33. Another example is given in the next section.

\section{The Wilf Polynomial}

In the Hardy-Ramanujan-Rademacher formula for $p(n)$ there occurs the factor

$$
A(k, n)=\sum_{(h, k)=1} \exp (\pi i s(h, k)-2 \pi i h n / k) .
$$

H. Wilf got the idea to use the $A(k, n)$ 's as roots of a polynomial

$$
Q(k, x)=\prod_{n=1}^{k}(x-A(k, n)) .
$$

Theorem 7.1. $Q(k, x)$ has integer coefficients if and only if $k$ is even or a square.

There are at least three different proofs [Almkvist 1994; Almkvist and Wilf 1995; Dokshitzer 1994]. Dokshitzer's proof is completely elementary.

There is a natural generalization

$$
Q(r, k, x)=\prod_{n=1}^{k}(x-A(r, k, n))
$$

Dokshitzer [1995] has proved the following result, again from scratch (see also [Almkvist 1994]):

Theorem 7.2. $Q(r, k, x)$ has integer coefficients if

$$
(r+1, k)=1 \quad \text { and } \quad(r+1, \varphi(k))=1,
$$

where $\varphi$ is Euler's totient function.

One gets the following byproduct [Almkvist 1994]:

Irregularity Condition for Pedestrians. A prime $p$ is irregular if and only if the integer

$$
\sum_{j=1}^{p-1} \cot ^{(r)}\left(\frac{j \pi}{p}\right) \cot \left(\frac{j \pi}{p}\right)
$$

is divisible by $p$ for some even $r \leq p-5$.

The usefulness of this criterion is limited by the size of the numbers. Thus for the smallest irregular prime $p=37$ we have

$$
\begin{aligned}
& \sum_{j=1}^{36} \cot ^{(30)}\left(\frac{j \pi}{37}\right) \cot \left(\frac{j \pi}{37}\right) \\
&= 99381121795017318051126492061551734 \backslash \\
& 87300616128232760119991858102272 \\
&=2^{32} \cdot 37 \cdot 6253775825561484753074079 \backslash \\
& 9068669445506250587268309366411 .
\end{aligned}
$$

\section{METHOD D: EISENSTEIN SERIES}

We try to compute the 65-digit number

$$
\begin{gathered}
a(2,300)=296884039333162678753061839296 \backslash \\
19499144044768568754234235191279016 .
\end{gathered}
$$

Using the asymptotic formula in Section 7 we obtain, taking 16 terms,

$$
\begin{gathered}
\sum_{j=1}^{16} \varphi_{j}(300)=296884039333162678753061839296 \backslash \\
19499144044768568841341805467003154.4927 .
\end{gathered}
$$

We get an error "already" in the 47th digit. (There is nothing wrong with the size of the terms: $\left|\varphi_{15}\right|<$ $\frac{1}{10}$.) The error is 87107570275724138.4927 . How can we get rid of such a large error? Let's go back to the beginning. We have

$$
f(x)=\prod_{\nu=1}^{\infty}\left(1-x^{\nu}\right)^{-\nu^{r}}=\sum_{0}^{\infty} a(r, n) x^{n}
$$


and

$$
\begin{aligned}
g(t) & =\log f(\exp (2 \pi i h / k-t)) \\
& =\sum_{\mu=1}^{\infty} \sum_{\nu=1}^{\infty} \frac{\nu^{r}}{\mu} \exp (\mu \nu(2 \pi i h / k-t)) .
\end{aligned}
$$

Take the derivative

$$
\begin{aligned}
g^{\prime}(t) & =-\sum_{\mu=1}^{\infty} \sum_{\nu=1}^{\infty} \nu^{r+1} \exp (-\mu \nu(t-2 \pi i h / k)) \\
& =-\sum_{n=1}^{\infty} \sigma_{r+1}(n) \exp (-n(t-2 \pi i h / k))
\end{aligned}
$$

where

$$
\sigma_{r+1}(n)=\sum_{d \mid n} d^{r+1}
$$

This we recognize as an Eisenstein series of weight $(r+2) / 2$. Indeed let

$$
G_{2 k}(\tau)=\sum_{m} \sum_{n}^{\prime}(m+n \tau)^{-2 k}
$$

for $k \geq 2$, where the sum is over all pairs of integers $\neq(0,0)$. The Fourier expansion of $G_{2 k}(\tau)$ is (see [Serre 1977, p. 150])

$$
G_{2 k}(\tau)=2 \zeta(2 k)+\frac{2(2 \pi i)^{2 k}}{(2 k-1) !} \sum_{n=1}^{\infty} \sigma_{2 k-1}(n) q^{n}
$$

where

$$
q=\exp (2 \pi i \tau), \quad \operatorname{Im} \tau>0 .
$$

Now by modularity

$$
G_{2 k}\left(\frac{a \tau+b}{c \tau+d}\right)=(c \tau+d)^{2 k} G_{2 k}(\tau)
$$

if $\left(\begin{array}{l}a b \\ c d\end{array}\right)$ is an integer matrix with $a d-b c=1$. We take

$$
\left(\begin{array}{ll}
a & b \\
c & d
\end{array}\right)=\left(\begin{array}{cc}
H & -(1+h H) / k \\
k & -h
\end{array}\right)
$$

where

$$
H h \equiv-1(\bmod k) .
$$

We have $q=\exp (2 \pi i \tau)=\exp (2 \pi i h / k-t)$, that is,

$$
\tau=h / k+i t / 2 \pi
$$

Then

$$
\tau^{\prime}=\frac{a \tau+b}{c \tau+d}=H / k+\frac{2 \pi i}{k^{2} t}
$$

and

$$
q^{\prime}=\exp \left(2 \pi i \tau^{\prime}\right)=\exp \left(2 \pi i H / k-\frac{4 \pi^{2}}{k^{2} t}\right) .
$$

Also

$$
c \tau+d=\frac{i k t}{2 \pi} .
$$

It follows that

$$
\begin{aligned}
g^{\prime}(t) & \\
= & -\sum_{n=1}^{\infty} \sigma_{r+1}(n) q^{n} \\
= & \frac{(r+1) ! \zeta(r+2)}{(2 \pi i)^{r+2}}-\frac{(r+1) !}{2(2 \pi i)^{r+2}} G_{r+2}(\tau) \\
= & \frac{\zeta(-1-r)}{2}-\left(\frac{i k t}{2 \pi}\right)^{-(r+2)} \frac{(r+1) !}{2(2 \pi i)^{r+2}} G_{r+2}\left(\frac{H}{k}+\frac{2 \pi i}{k^{2} t}\right) \\
= & \frac{\zeta(-1-r)}{2}-\frac{(r+1) ! \zeta(r+2)}{k^{r+2} t^{r+2}} \\
& -\frac{(2 \pi i)^{r+2}}{(k t)^{r+2}} \sum_{n=1}^{\infty} \sigma_{r+1}(n) \exp \left(\frac{2 \pi i n H}{k}\right) \exp \left(-\frac{4 \pi^{2} n}{k^{2} t}\right) .
\end{aligned}
$$

Integrating, we obain the following formula (we know the constant from the Mellin transformation)

$g(t)$

$$
\begin{array}{r}
=\frac{r ! \zeta(r+2)}{k^{r+2}} \frac{1}{t^{r+1}}+\frac{\zeta(-1-r)}{2} t+\pi i s(r, h, k)+k^{r} \zeta^{\prime}(-r) \\
-\frac{(2 \pi i)^{r+2} k^{r}}{4 \pi^{2}} \sum_{n=1}^{\infty} \frac{\sigma_{r+1}(n)}{n} \exp \left(\frac{2 \pi i H n}{k}\right) \exp \left(-\frac{4 \pi^{2} n}{k^{2} t}\right) \\
\left(\left(k^{2} t\right)^{-r}+\sum_{\nu=1}^{r} \nu !\left(\begin{array}{l}
r \\
\nu
\end{array}\right)\left(4 \pi^{2} n\right)^{-\nu}\left(k^{2} t\right)^{\nu-r}\right) .
\end{array}
$$

This agrees with the result we got from the Mellin transformation up to the last term. When $t \rightarrow 0+$ this term is very small.

Now we want to estimate the error caused by the last term. We specialize to $r=2$ and put

$$
a=2 \zeta(4)=\pi^{4} / 45, \quad b=4 \pi^{2} .
$$


Then

$$
\begin{aligned}
& g(t)=\frac{a}{k^{4}} \frac{1}{t^{3}}+\frac{t}{240}+\pi i s(2,h, k)+k^{2} \zeta^{\prime}(-2) \\
&-k^{2} b \sum_{\nu=1}^{\infty} \frac{\sigma_{3}(\nu)}{\nu} \exp \left(\frac{2 \pi i \nu H}{k}\right) \exp \left(-\frac{\nu b}{k^{2} t}\right) \\
& \times\left(\frac{t^{-2}}{k^{4}}+\frac{2 t^{-1}}{\nu b k^{2}}+\frac{2}{\nu b^{2}}\right) .
\end{aligned}
$$

Put

$$
c=\exp \zeta^{\prime}(-2), \quad \xi=n+\frac{1}{240} .
$$

Computing $f(\exp (2 \pi i h / k-t))=\exp (g(t))$, we take only $\nu=1$ in the last term. We will get the pseudodifferential operator

$-\exp \left(\frac{2 \pi i H}{k}\right) \exp \left(-\frac{b D^{-1}}{k^{2}}\right)\left(\frac{b}{k^{2}} D^{-2}+2 D^{-1}+\frac{2 k^{2}}{b}\right)$

(where $D=\frac{d}{d \xi}$ ) acting on the $\varphi_{k}(n)$-term.

Let's find (formally)

$$
\begin{aligned}
D^{-m} \exp \left(-c D^{-1}\right) \xi^{\mu} & =\sum_{j=0}^{\infty} \frac{(-1)^{j} c^{j}}{j !} D^{-j-m} \xi^{\mu} \\
& =\mu ! \sum_{j=0}^{\infty} \frac{(-1)^{j} c^{j}}{j !(j+m+\mu) !} \xi^{\mu+j+m} \\
& =\mu !\left(\frac{\xi}{c}\right)^{\frac{m+\mu}{2}} J_{m+\mu}(2 \sqrt{c \xi})
\end{aligned}
$$

where $J_{m+\mu}(x)$ is the Bessel function.

Then (abusing the notation in first line)

$$
\begin{aligned}
& \sum_{(h, k)=1} \exp (2 \pi i H / k) D^{-m} \exp \left(-\frac{b D^{-1}}{k^{2}}\right) \varphi_{k}(n) \\
&=\tilde{A}(k, n) a c^{k^{2}} k^{m-2}\left(\frac{\xi}{b}\right)^{1+m / 2} \sum_{\nu=0}^{\infty} \frac{1}{(\nu+1) !} \\
& \times\left(\frac{a}{k}\left(\frac{\xi}{b}\right)^{3 / 2}\right)^{\nu} J_{3 \nu+m+2}\left(\frac{2 \sqrt{b \xi}}{k}\right),
\end{aligned}
$$

where

$$
\tilde{A}(k, n)=\sum_{(h, k)=1} \exp \left(\pi i s(2, h, k)+\frac{2 \pi i H}{k}-\frac{2 \pi i h n}{k}\right) .
$$

For each $k=1,2,3, \ldots$ we get three terms (corresponding to $m=0,1,2)$.
The largest for $n=300$ are:

$$
\begin{aligned}
& \underline{k=1} \quad m=2 \quad-85757379661963506.1407 \\
& m=1 \quad-1339472453656529.1035 \\
& m=0 \quad 10448434935559.0223 \\
& \underline{k=2} \quad m=2 \quad 265998816.6964 \\
& m=1 \quad 8298988.6074 \\
& m=0 \quad 129052.9901 \\
& \underline{k=3} \quad m=2 \quad 376093.7907 \\
& m=1 \quad 17575.3790 \\
& m=0 \quad 408.7188 \\
& \underline{k=4} \quad m=2 \quad 6932.2282 \\
& m=1 \quad 431.3693 \\
& m=0 \quad 13.3356 \\
& \underline{k=5} \quad m=2 \quad-97.1363
\end{aligned}
$$

Summing up we get

$-87107570275727378.2885$

which is very close to the error we got when we used the Mellin transformation. The remaining error is only -3240 .

If we expand $\exp (g(t))$ further (for $\nu=1$ ) we get a second term

$$
\exp \left(-2 b D^{-1}\right)\left(\frac{b^{2}}{2} D^{-4}+2 b D^{-3}+\cdots\right)
$$

If we let this act on $\varphi_{1}$ we get for

$$
\begin{array}{lc}
m=4 & -185.8528, \\
m=3 & -0.3 .
\end{array}
$$

There is a lot of cancellation. In the last sum there are terms of order $10^{8}$ but the sum is -0.3 .

\section{PLANE PARTITIONS, THE FIFTH AND SIXTH TERMS}

For plane partitions we have $r=1$, so

$$
f(x)=\prod_{\nu=1}^{\infty}\left(1-x^{\nu}\right)^{-\nu}
$$

and we get a Mellin transform 
$\log f(\exp (2 \pi i h / k-t))$

$$
\begin{aligned}
= & \frac{\zeta(3)}{k^{3}} \frac{1}{t^{2}}+k \zeta^{\prime}(-1) \\
& +\frac{k \log k}{12}+\frac{k}{2} \sum_{j=1}^{k-1} B_{2}(j / k) \log \left|2 \sin \left(\frac{j h \pi}{k}\right)\right| \\
& +\frac{k}{12} \log t+\frac{i k^{2} t}{6} \sum_{j=1}^{k-1} B_{3}(j / k) \cot \left(\frac{j h \pi}{k}\right) \\
& +\sum_{\nu=2}^{\infty} \frac{(-1)^{\nu} k^{\nu+1} t^{\nu}}{\nu(\nu+2) \nu !}\left(B_{\nu} B_{\nu+2}\right. \\
& \left.+\frac{\nu}{(2 i)^{\nu}} \sum_{j=1}^{k-1} B_{\nu+2}(j / k) \cot ^{(\nu-1)}\left(\frac{j h \pi}{k}\right)\right) .
\end{aligned}
$$

For $k=1,2,3,4$ the computations in [Almkvist 1993] are correct, so let's take $k=5$. After some computations we get (taking terms only up to $D^{3}$ )

$$
\begin{aligned}
\varphi_{5}(n) \sim & 2 c^{5}\left(\frac{a}{125}\right)^{17 / 24} \sum_{j=1}^{2} w_{j} \exp \left(b_{j} D^{2}\right) \\
& \times \cos \left(\frac{2 \pi j n}{5}+a_{j} D+c_{j} D^{3}\right) g\left(n \sqrt{\frac{a}{125}},-\frac{5}{12}\right),
\end{aligned}
$$

where

$$
\begin{aligned}
D & =\frac{d}{d n}, \\
a & =\zeta(3), \\
c & =\exp \left(\zeta^{\prime}(-1)\right), \\
w_{1} & =5^{5 / 12}(2 \sin (\pi / 5))^{1 / 30}(2 \sin (2 \pi / 5))^{-11 / 30}, \\
w_{2} & =5^{5 / 12}(2 \sin (2 \pi / 5))^{1 / 30}(2 \sin (\pi / 5))^{-11 / 30}, \\
a_{1} & =\frac{1}{5}(2 \cot (\pi / 5)+\cot (2 \pi / 5)), \\
a_{2} & =\frac{1}{5}(2 \cot (2 \pi / 5)+\cot (4 \pi / 5)), \\
b_{1} & =\frac{619}{2880}-\frac{\sqrt{5}}{10}, \\
b_{2} & =\frac{619}{2880}+\frac{\sqrt{5}}{10}, \\
c_{1} & =\frac{1}{600}\left(74 \frac{\cot ^{2}(\pi / 5)}{\sin ^{2}(\pi / 5)}+43 \frac{\cot ^{2}(2 \pi / 5)}{\sin ^{2}(2 \pi / 5)}\right),
\end{aligned}
$$

$$
\begin{aligned}
c_{2} & =\frac{1}{600}\left(74 \frac{\cot (2 \pi / 5)}{\sin ^{2}(2 \pi / 5)}+43 \frac{\cot (4 \pi / 5)}{\sin ^{2}(4 \pi / 5)}\right) \\
g(x, \gamma) & =\sum_{\nu=0}^{\infty} \frac{x^{2 \nu+\gamma-1}}{\nu ! \Gamma(2 \nu+\gamma)} .
\end{aligned}
$$

For $k=6$, which is a much simpler case, we get

$$
\begin{aligned}
& \begin{aligned}
\varphi_{6}(n) \sim & c^{6} 2^{1 / 4} 3^{1 / 3}\left(\frac{a}{216}\right)^{3 / 4} \\
\times & \left(2 \sin \frac{2 \pi n}{6}\left(\frac{19}{9 \sqrt{3}} D+\frac{10503461}{2099520 \sqrt{3}} D^{3}+\cdots\right)\right. \\
& \left.+2 \cos \frac{2 \pi n}{6}\left(1-\frac{84787}{77760} D^{2}-\cdots\right)\right) g\left(n \sqrt{\frac{a}{216}},-\frac{1}{2}\right),
\end{aligned} \\
& \text { with } D=\frac{d}{d n} .
\end{aligned}
$$

Numerical Example. Let $n=199$. Then

$$
\varphi_{5}=-720.6, \quad \varphi_{6}=35.7 .
$$

If $n=200$ we get

$$
\varphi_{5}=2549.6 \quad \varphi_{6}=-32.1 .
$$

For $\varphi_{1}, \ldots, \varphi_{4}$, see [Almkvist 1993, p. 24]. The errors will be 47 and 4 respectively; $\pi(199)$ and $\pi(200)$ are numbers with 28 digits.

\section{TRIANGULAR PARTITIONS}

Consider a triangular array $T_{r}$ of nonnegative integers $a_{i j}$ :

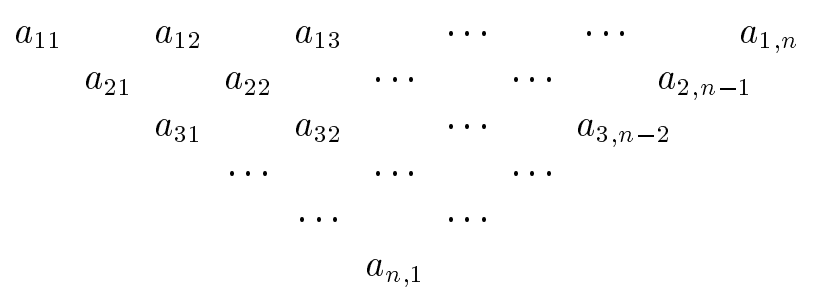

such that

$$
a_{i j} \geq a_{i+1, j} \quad \text { and } \quad a_{i j} \geq a_{i+1, j-1} .
$$

If $\sum_{i+j \leq r+1} a_{i j}=n$ we say that $T_{r}$ is a triangular partition of $n$ of order $r$. Let $T_{r}(n)$ be the number of such partitions of $n$ of order $r$. 
Carlitz and Scoville [1975] found the generating function

$$
f_{r}(x)=\sum_{n=0}^{\infty} T_{r}(n) x^{n}=\prod_{\nu=1}^{r}\left(1-x^{2 \nu-1}\right)^{\nu-r-1} .
$$

Since $f_{r}(x)$ is the inverse of a polynomial, $T_{r}(n)$ will have polynomial growth in $n$. More precisely, $T_{r}(n)$ is a quasipolynomial, i.e., its coefficients are periodic functions of $n$.

We compute (for odd $k \leq 2 r-1$ )

$$
\begin{aligned}
& g(t)=\log f_{r}(\exp (2 \pi i h / k-t))=\sum_{\nu=1}^{r} \sum_{\mu=0}^{\infty} \frac{r+1-\mu}{\mu} \exp (2 \pi i h \mu(2 \nu-1) / k) \exp (-\mu(2 \nu-1) t), \\
& \tilde{g}(s)=\int_{0}^{\infty} g(t) t^{s-1} d t=\Gamma(s) k^{-1-s} \sum_{\nu=1}^{r} \frac{r+1-\nu}{(2 \nu-1)^{s}} \sum_{d=1}^{k} \zeta(1+s, d / k) \exp (2 \pi i h d(2 \nu-1) / k) .
\end{aligned}
$$

The computation of the residues at the poles is complicated. We specialize to $r=4$ and give only the final result

$$
T_{4}(n)=\varphi_{1}(n)+\varphi_{3}(n)+\varphi_{5}(n)+\varphi_{7}(n),
$$

where (with $\xi=n+15$ )

$$
\begin{aligned}
& \varphi_{1}(n)=\frac{1}{3^{3} 5^{2} 7}\left(\frac{\xi^{9}}{9 !}-\frac{65}{12} \frac{\xi^{7}}{7 !}+\frac{11537}{720} \frac{\xi^{5}}{5 !}+\frac{3881}{112} \frac{\xi^{3}}{3 !}+\frac{500819}{8100} \xi\right), \\
& \varphi_{3}(n)=3^{-13 / 2}\left(\xi^{2}-\frac{110}{3}\right) \sin \frac{2 \pi n}{3}+3^{-7} \xi \cos \frac{2 \pi n}{3} \\
& \varphi_{5}(n)=-\frac{\xi}{625}\left(2 \cos \frac{2 n \pi}{5}+2 \cos \frac{4 n \pi}{5}\right)-\frac{1}{625}\left(2 \sin \frac{2 n \pi}{5}\left(2 \cot \frac{\pi}{5}-\cot \frac{2 \pi}{5}\right)+2 \sin \frac{4 n \pi}{5}\left(\cot \frac{\pi}{5}+2 \cot \frac{2 \pi}{5}\right)\right), \\
& \varphi_{7}(n)=\frac{1}{196}\left(\frac{\sin \frac{(n+1) \pi}{7}}{\sin ^{2} \frac{3 \pi}{7} \sin \frac{5 \pi}{7}}+\frac{\sin \frac{3(n+1) \pi}{7}}{\sin ^{2} \frac{5 \pi}{7} \sin \frac{\pi}{7}}-\frac{\sin \frac{5(n+1) \pi}{7}}{\sin ^{2} \frac{\pi}{7} \sin \frac{3 \pi}{7}}\right) .
\end{aligned}
$$

The expressions in outer parentheses are all integers. We obtain an exact formula.

Numerical Example. Let $r=4$ and $n=998$. Then

$$
\begin{array}{rr}
\varphi_{1}= & 654870000064421794.362848, \\
\varphi_{3}= & -704.026063, \\
\varphi_{5}= & 1.622400, \\
\varphi_{7}= & 0.040816, \\
\varphi_{1}+\varphi_{3}+\varphi_{5}+\varphi_{7}= & 654870000064421092.000001,
\end{array}
$$

which agrees with $T_{4}(998)$. 


\section{THE LONG HISTORY OF KINKELIN'S CONSTANT}

Kinkelin [1860] generalized the $\Gamma$-function to the function $\Gamma_{2}(x)$. In modern notation (see [Vardi 1988]) it satisfies

$$
\Gamma_{2}(n+2)=\frac{1}{1 ! \times 2 ! \times \cdots \times n !}
$$

if $n$ is a positive integer. There is an asymptotic formula, similar to Stirling's formula for $\Gamma$ :

$\log \Gamma_{2}(x)=\frac{3}{4} x^{2}-\left(\frac{1}{2} x^{2}-\frac{1}{12}\right) \log x-\frac{1}{2} x \log (2 \pi)$

$$
-K+O\left(x^{-1}\right)
$$

where $K$ is Kinkelin's constant. The corresponding constant for the $\Gamma$-function is

$$
-\frac{1}{2} \log (2 \pi)=\zeta^{\prime}(0)
$$

In Kinkelin's notation, $K=\frac{1}{2} \log \tilde{\omega}$.

The constant $K$ has been rediscovered a number of times and we give several formulas for it.

From [Kinkelin 1860; Knessl 1990] we have

$$
\begin{aligned}
K=-\lim _{N \rightarrow \infty}( & \sum_{1}^{N} n \log n-\frac{N^{2}}{2} \log N+\frac{N^{2}}{4} \\
& \left.-N \log N-\frac{1}{12} \log N-\frac{1}{12}\right) .
\end{aligned}
$$

Knessl found his formula when using WKB-approximation for $\pi(n)$.

From [Wright 1931]:

$$
K=2 \int_{0}^{\infty} \frac{x \log x}{e^{2 \pi x}-1} d x .
$$

This is Wright's constant in his asymptotic formula for $\pi(n)$.

From [Kinkelin 1860]:

$$
\begin{aligned}
K= & \frac{1}{24}-\frac{1}{3} \gamma-\sum_{\nu=1}^{\infty} \frac{\zeta(2 \nu+1)-1}{(2 \nu+1)(2 \nu+3)} \\
K= & \frac{1}{36}(\gamma-2)-\log 4+\frac{1}{9}(3 \log 3+5 \log 5) \\
& +\frac{1}{9} \sum_{\nu=1}^{\infty} \frac{\zeta(2 \nu+1)-1}{(\nu+1)(2 \nu+1)} 4^{-(2 \nu+1)}
\end{aligned}
$$

$$
K=\frac{1}{12}-\frac{1}{4} \log (2 \pi)+\int_{0}^{1} x \Gamma(x) d x .
$$

The latter series converges very fast and 60 terms will give 48 correct digits. Kinkelin computed $K$ with 8 correct digits.

In $1990 \mathrm{I}$ found the formulas

$$
\begin{aligned}
& K=-\frac{1}{12} \gamma+\log 2-\frac{5}{6}+\frac{1}{2} \sum_{\nu=2}^{\infty} \frac{(-1)^{\nu}(\zeta(\nu)-1)}{(\nu+1)(\nu+2)}, \\
& K=\frac{1}{12}\left(1-\gamma-\log 2 \pi+\frac{6}{\pi^{2}} \zeta^{\prime}(2)\right) \\
& K=-\frac{1}{6}\left(1+\sum_{\nu=3}^{\infty} \frac{B_{\nu}}{\left(\begin{array}{c}
\nu \\
3
\end{array}\right)}\right)
\end{aligned}
$$

The last series is divergent but if we stop at $\nu=10$ we get $K=-0.16548$ with an error of 0.00006 .

From [Vardi 1988] we have

$$
K=\zeta^{\prime}(-1)
$$

When computing the approximations of $\pi(n)$ we need $K=\zeta^{\prime}(-1)$ with many digits. Cheema and Conway [1972] computed Wright's integral but only with 14 correct digits.

For even positive $r$ one can use the formula

$$
\zeta^{\prime}(-r)=(-1)^{r / 2} \pi r ! \zeta(r+1)(2 \pi)^{-r-1} .
$$

Otherwise one uses the approximation

$$
f^{\prime}(x) \approx \frac{f(x+h)-f(x-h)}{2 h}
$$

with $h=10^{-n / 2}$. Compute with $3 n / 2$ digits if you want $n$ digits.

\section{CONCLUSION}

The methods mentioned here (the Mellin transformation and the Ramanujan-type computation) can be used to find various asymptotic formulas. We mention some cases that have been treated. 
1. Partitions into parts of size at least $r$ [Dixmier and Nicolas 1990]:

$$
\prod_{\nu=r}^{\infty}\left(1-x^{\nu}\right)^{-1}
$$

2. Partitions into parts of size at most $r$ :

$$
\prod_{\nu=1}^{r}\left(1-x^{\nu}\right)^{-1}
$$

3. Partitions into distinct parts of size at least $r$ [Dixmier and Nicolas 1990]:

$$
\prod_{\nu=r}^{\infty}\left(1+x^{\nu}\right)
$$

4. Partitions into distinct odd parts of size at least $2 r-1$ :

$$
\prod_{\nu=2 r-1}^{\infty}\left(1+x^{2 \nu-1}\right)
$$

5. Partitions into $p$-cores [Garvan 1993]:

$$
\prod_{\nu=1}^{\infty} \frac{\left(1-x^{p \nu}\right)^{p}}{1-x^{\nu}}
$$

6.

$$
\prod_{\substack{m, n \in \mathbb{Z} \\(m, n) \neq(0,0)}}\left(1-x^{m^{2}+7 n^{2}}\right)^{-1}
$$

7. [Fee and Granville 1991]

$$
\prod_{\nu=1}^{\infty}\left(1-x^{\nu}\right)^{\mu(\nu)}=\sum_{0}^{\infty} a(n) x^{n}
$$

In an unpublished paper with Meurman the following asymptotic formula is found:

$$
a(n) \sim \cos (n \pi / 3+\pi / 4) \exp \left(0.4377 \log ^{3} n+\cdots\right)
$$

for $n<10^{12}$. The real asymptotic behaviour of $a(n)$ does not occur until $n>10^{35}$ and is unknown.

It is the author's hope that the computations made in this paper can be justified and, at least in some cases, the errors can be estimated.

\section{ACKNOWLEDGEMENTS}

I want to thank George Andrews, Bruce Berndt, Tim Dokshitzer, Shalosh B. Ekhad, Petr Lisonek, Herb Wilf and Doron Zeilberger for assistance in various ways. Above all my thanks go to Arne Meurman who has contributed in various ways, through discussions and making computations.

\section{REFERENCES}

[Almkvist 1993] G. Almkvist, "A rather exact formula for the number of plane partitions", pp. 21-26 in $A$ tribute to Emil Grosswald: number theory and related analysis, edited by M. Knopp and M. Sheingorn, Contemp. Math. 143, Amer. Math. Soc., Providence, RI, 1993.

[Almkvist 1994] G. Almkvist, "Wilf's conjecture and a generalization", pp. 211-233 in The Rademacher legacy to mathematics (University Park, PA, 1992), edited by G. E. Andrews et al., Contemp. Math. 166, Amer. Math. Soc., Providence, RI, 1994.

[Almkvist and Wilf 1995] G. Almkvist and H. S. Wilf, "On the coefficients in the Hardy-RamanujanRademacher formula for $p(n)$ ", J. Number Theory 50:2 (1995), 329-334.

[Andrews 1976] G. E. Andrews, The theory of partitions, Encyclopedia of Mathematics and its Applications, Addison-Wesley, Reading, MA, 1976.

[Carlitz and Scoville 1975] L. Carlitz and R. Scoville, "A generating function for triangular partitions", Math. Comp. 29 (1975), 67-77.

[Cheema and Conway 1972] M. S. Cheema and W. E. Conway, "Numerical investigation of certain asymptotic results in the theory of partitions", Math. Comp. 26 (1972), 999-1005. Addendum, 26 (1972), no. 120 , loose microfiche suppl. C1-C10.

[Dixmier and Nicolas 1990] J. Dixmier and J.-L. Nicolas, "Partitions without small parts", pp. 9-33 in Number theory I (Budapest, 1987), edited by K. Győry et al., Colloq. Math. Soc. János Bolyai 51, North-Holland, Amsterdam, 1990.

[Dokshitzer 1994] T. Dokshitzer, "An elementary proof of Wilf's conjecture", pp. 235-243 in The Rademacher legacy to mathematics (University Park, PA, 1992), edited by G. E. Andrews et al., Contemp. Math. 166, Amer. Math. Soc., Providence, RI, 1994. 
[Dokshitzer 1995] T. Dokshitzer, "On Wilf's conjecture and generalizations", pp. 133-153 in Number theory (Halifax, Canada, 1994), edited by K. Dilcher, CMS Conf. Proc. 15, Amer. Math. Soc., Providence, RI, 1995.

[Fee and Granville 1991] G. Fee and A. Granville, "The prime factors of Wendt's binomial circulant determinant", Math. Comp. 57:196 (1991), 839-848.

[Garvan 1993] F. G. Garvan, "Some congruences for partitions that are $p$-cores", Proc. London Math. Soc. (3) 66:3 (1993), 449-478.

[Henrici 1974] P. Henrici, Applied and computational complex analysis, 1: Power series, integration, conformal mapping, location of zeros, WileyInterscience, New York, 1974.

[Kinkelin 1860] J. Kinkelin, "Über eine mit der Gammafunktion verwandte Transcendente und deren Anwendung auf die Integralrechnung", J. Reine und Angew. Math 57 (1860), 122-158.

[Knessl 1990] C. Knessl, "Asymptotic behaviour of high order differences of the plane partition function", Preprint, Univ. of Illinois at Chicago, 1990.

[Mellin 1923] H. Mellin, Anwendung einer allgemeinen Methode zur Herleitung asymptotisher Formeln, Suomalaisen Tiedeakatemian, Helsinki, 1923.
[Milnor 1983] J. Milnor, "On polylogarithms, Hurwitz zeta functions, and the Kubert identities", Enseign. Math. (2) 29:3-4 (1983), 281-322.

[Rademacher 1933] H. Rademacher, "Egy reciprocitásképletről a modelfüggvények elméletéből", Math. Fiz. Lapok 40 (1933), 24-34.

[Rademacher and Grosswald 1972] H. Rademacher and E. Grosswald, Dedekind sums, Carus Mathematical Monographs 16, The Mathematical Association of America, Washington, DC, 1972.

[Serre 1977] J.-P. Serre, Cours d'arithmétique, 2nd ed., Presses Universitaires de France, Paris, 1977. First edition translated as A course in Arithmetic, Springer, 1973.

[Vardi 1988] I. Vardi, "Determinants of Laplacians and multiple gamma functions", SIAM J. Math. Anal. 19:2 (1988), 493-507.

[Wright 1931] E. M. Wright, "Asymptotic partition formulæ, I: Plane partitions", Quart. J. Math. 2 (1931), 177-189.

[Zagier 1986] D. Zagier, "Hyperbolic manifolds and special values of Dedekind zeta-functions", Invent. Math. 83:2 (1986), 285-301.

[Zucker and Robertson 1975] I. J. Zucker and M. M. Robertson, "Exact values for some two-dimensional lattice sums", J. Phys. A 8 (1975), 874-881.

Gert Almkvist, Matematiska Institutionen, Lunds Universitet, Lund, Sweden (gert@maths.lth.se)

Received July 2, 1996; accepted in revised form March 30, 1998 